\title{
Communication \\ Development of a 1 TW/35 fs Ti:sapphire Laser Amplifier and Generation of Intense THz Waves Using Two-Color Laser Filamentation
}

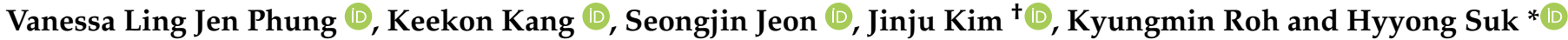 \\ Department of Physics and Photon Science, Gwangju Institute of Science and Technology, Buk-gu, \\ Gwangju 61005, Korea; vplj@gist.ac.kr (V.L.J.P.); keekon@gist.ac.kr (K.K.); anffldhkd418@gist.ac.kr (S.J.); \\ pearl2kim@gist.ac.kr (J.K.); 20161077@gist.ac.kr (K.R.) \\ * Correspondence: hysuk@gist.ac.kr \\ + Current address: Advanced Photonics Research Institute (APRI), Gwangju Institute of Science and Technology, \\ Buk-gu, Gwangju 61005, Korea.
}

check for updates

Citation: Phung, V.L.J.; Kang, K.; Jeon, S.; Kim, J.; Roh, K.; Suk, H. Development of a $1 \mathrm{TW} / 35 \mathrm{fs}$ Ti:sapphire Laser Amplifier and Generation of Intense $\mathrm{THz}$ Waves Using Two-Color Laser Filamentation. Photonics 2021, 8, 316. https:// doi.org/10.3390/photonics 8080316

Received: 24 June 2021

Accepted: 2 August 2021

Published: 5 August 2021

Publisher's Note: MDPI stays neutral with regard to jurisdictional claims in published maps and institutional affiliations.

Copyright: (c) 2021 by the authors. Licensee MDPI, Basel, Switzerland. This article is an open access article distributed under the terms and conditions of the Creative Commons Attribution (CC BY) license (https:// creativecommons.org/licenses/by/ $4.0 /)$

\begin{abstract}
We developed a compact Ti:sapphire laser amplifier system in our laboratory, generating intense laser pulses with a peak power of $>1 \mathrm{TW}$ (terawatt), a pulse duration of $34 \mathrm{fs}$ (femtosecond), a central wavelength of $800 \mathrm{~nm}$, and a repetition rate of $10 \mathrm{~Hz}$. The laser amplifier system consists of a mode-locked Ti:sapphire oscillator, a regenerative amplifier, and a single-side-pumped 4-pass amplifier. The chirped-pulse amplification (CPA)-based laser amplifier was found to provide an energy of $49.6 \mathrm{~mJ}$ after compression by gratings in air, where the pumping fluence of $1.88 \mathrm{~J} / \mathrm{cm}^{2}$ was used. The amplified spontaneous emission (ASE) level was measured to be lower than $10^{-7}$, and ps-prepulses were in $10^{-4}$ or lower level. The developed laser amplifier system was used for the generation of intense $\mathrm{THz}$ (terahertz) waves by focusing the original $(800 \mathrm{~nm})$ and second harmonic $(400 \mathrm{~nm})$ laser pulses in air. The THz pulse energy was shown to be saturated in the high laser energy regime, and this phenomenon was confirmed by fully electromagnetic, relativistic, and self-consistent particle-in-cell (PIC) simulations.
\end{abstract}

Keywords: Ti:sapphire laser; laser amplifier; fs CPA laser; high power laser; $\mathrm{THz}$

\section{Introduction}

Since the demonstration of the first laser in 1960 [1], extensive efforts have been made to develop lasers with higher power/energy and shorter pulses [2]. A breakthrough in laser power was made by the invention of the chirped-pulse-amplification (CPA) technique in 1985 [3], and since then, many TW laser systems and even PW laser systems have been developed, achieving a maximum focused intensity of $5.5 \times 10^{22} \mathrm{~W} / \mathrm{cm}^{2}[4,5]$. Thus far, most of them have been used for laser-plasma interaction studies, including high-energy electron acceleration [6-8], ion generation [9,10], X-ray/ $\gamma$-ray production $[11,12]$, etc. In our laboratory at GIST (Gwangju Institute of Science and Technology), we also have a 20 TW/35 fs Ti:sapphire laser system that is used mainly for high-energy electron acceleration and related experiments. However, there is also a demand for lower power lasers of $\sim 1$ TW and a higher repetition rate for other researches. For example, they can be used for the generation of intense $\mathrm{THz}$ wave pulses [13], fs electron beam pulses for ultrafast electron diffraction (UED) [14], X-ray pulses for time-resolved X-ray crystallography and absorption spectroscopy $[15,16]$, etc. Hence, we developed a 1 TW-class Ti:sapphire amplifier using some parts of the existing 20 TW laser system, allowing a separate beam line for other researches. To build the separate laser beam line, we added a single-side-pumped 4-pass amplifier and an in-air pulse compressor to the existing oscillator and regenerative amplifier, leading to a separate 1 TW laser system. The developed laser system turned out to have a very good performance, for example, reaching an ASE level of less than $10^{-7}$. The laser amplifier system has ps-prepulses, but their intensities are in $10^{-4}$ or lower levels. 
The prepulse effect is not important in our laser-plasma-based THz research as we need laser-produced plasma in gas. The Ti:sapphire amplifier system was designed and built by ourselves in-house, providing a unique laser facility for research.

We used the laser amplifier system to generate intense $\mathrm{THz}$ waves. Compared with other electromagnetic radiations, $\mathrm{THz}$ waves have some unique features. For example, they can interact strongly with chemical/biological molecules (in rotation and vibration modes). Furthermore, $\mathrm{THz}$ waves are reflected from conductors, but they can pass through dielectric materials easily. Based on these properties, $\mathrm{THz}$ waves have a wide range of important applications in science and technology, including THz imaging, spectroscopy, medical diagnostics, security, etc. $[17,18]$. To generate intense $\mathrm{THz}$ waves, we focused the original $(800 \mathrm{~nm})$ and second harmonic $(400 \mathrm{~nm})$ laser pulses in air, where an asymmetric net field in the plasma produces a strong $\mathrm{THz}$ pulse. Our experimental result shows that the $\mathrm{THz}$ pulse energy increases sharply in the beginning as the laser energy increases, but it is saturated eventually in the high laser energy regime. As far as we know, this saturation phenomenon has not been explained by any rigorous theory or simulation. Hence, we performed fully electromagnetic and self-consistent particle-in-cell (PIC) simulations to investigate this phenomenon and found that the PIC simulation result also leads to saturation in the $\mathrm{THz}$ energy, which is in agreement with the experimental result. In this paper, we will report details of the Ti:sapphire laser amplifier development and the experimental result for two-color-based intense $\mathrm{THz}$ wave generation together with the simulation results.

\section{Development of the $1 \mathrm{TW} / 35$ fs Ti:sapphire Laser Amplifier System}

\subsection{Overall Configuration}

Figure 1 illustrates the configuration of the Ti:sapphire laser amplifier system that was developed recently in our laboratory. It consists of a Ti:sapphire oscillator, a stretcher grating, a Ti:sapphire regenerative amplifier pumped by an Nd:YLF laser, a 4-pass Ti:sapphire amplifier, and a pulse compressor. The Kerr-lens mode-locked oscillator (FemtoLasers Produktions $\mathrm{GmbH}$, model Fusion 20) generates a train of broadband pulses at a repetition rate of $80 \mathrm{MHz}$, and the oscillator laser beam has a central wavelength of $796 \mathrm{~nm}$, an energy of $3 \mathrm{~nJ} /$ pulse, and a pulse duration of $20 \mathrm{fs}$. The oscillator pulse is stretched to $312 \mathrm{ps}$ (FWHM) by a grating with groove density 1100 lines $/ \mathrm{mm}$, and then it is injected into the regenerative amplifier (Spectra-Physics, model Spitfire Pro) that is pumped by a Q-switched Nd:YLF laser (Spectra-Physics, model Empower 30) with a wavelength of $527 \mathrm{~nm}$ and $20 \mathrm{~mJ} /$ pulse at $1 \mathrm{kHz}$. The laser pulse is trapped in the cavity of the regenerative amplifier and is amplified to $4 \mathrm{~mJ}$ over 12-15 round trips for enough energy, where two Pockels cells (PC1, PC2) are used for trapping and ejection of the laser pulse, as shown in Figure 1. After that, the laser beam from the regenerative amplifier is sent to another Pockels cell (PC3, model QX-1020 by Gooch and Housego) for pulse cleaning, and then it is injected into the in-house 4-pass amplifier for higher energy.

The 4-pass amplifier consists of a Titanium-doped sapphire crystal with a diameter of $15 \mathrm{~mm}$ and a length of $20 \mathrm{~mm}$, and it is single-side-pumped by the frequency-doubled (532 $\mathrm{nm}$ in wavelength) Nd:YAG laser (Continuum, model Surelite-EX) at $10 \mathrm{~Hz}$. The injected laser pulse in the 4-pass amplifier passes through the Ti:sapphire crystal four times for enough amplification. The pump beam diameter was reduced from $10 \mathrm{~mm}$ to $5 \mathrm{~mm}$ using a Galilean telescope consisting of a pair of convex and concave lenses to increase the pump beam fluence up to $1.88 \mathrm{~J} / \mathrm{cm}^{2}$. The stretched, amplified laser pulse is sent at an incidence angle of $56^{\circ}$, to the Treacy configuration pulse compressor [19], which consists of a pair of Au-coated, reflective gratings with groove density 1500 lines $/ \mathrm{mm}$ (produced by Spectrogon Inc.) and a vertical retro-reflector (VRR). To prevent potential damage on the gratings, especially from the compressed beam, the output laser beam from the 4-pass amplifier was expanded to $17 \mathrm{~mm}$ in diameter, resulting in a maximum energy fluence of $39 \mathrm{~mJ} / \mathrm{cm}^{2}$. This fluence will be adequate because the calculated damage threshold of the compressor grating is approximately $78 \mathrm{~mJ} / \mathrm{cm}^{2}(800 \mathrm{~nm}, 10 \mathrm{~Hz}, 35 \mathrm{fs})$. In this way, we developed a high-power Ti:sapphire laser amplifier system that can deliver laser pulses with an energy 
of $49.6 \mathrm{~mJ} /$ pulse and a pulse duration of $34 \mathrm{fs}$, leading to more than $1 \mathrm{TW}$ in peak power. More details for the development of the laser amplifier system are described below.

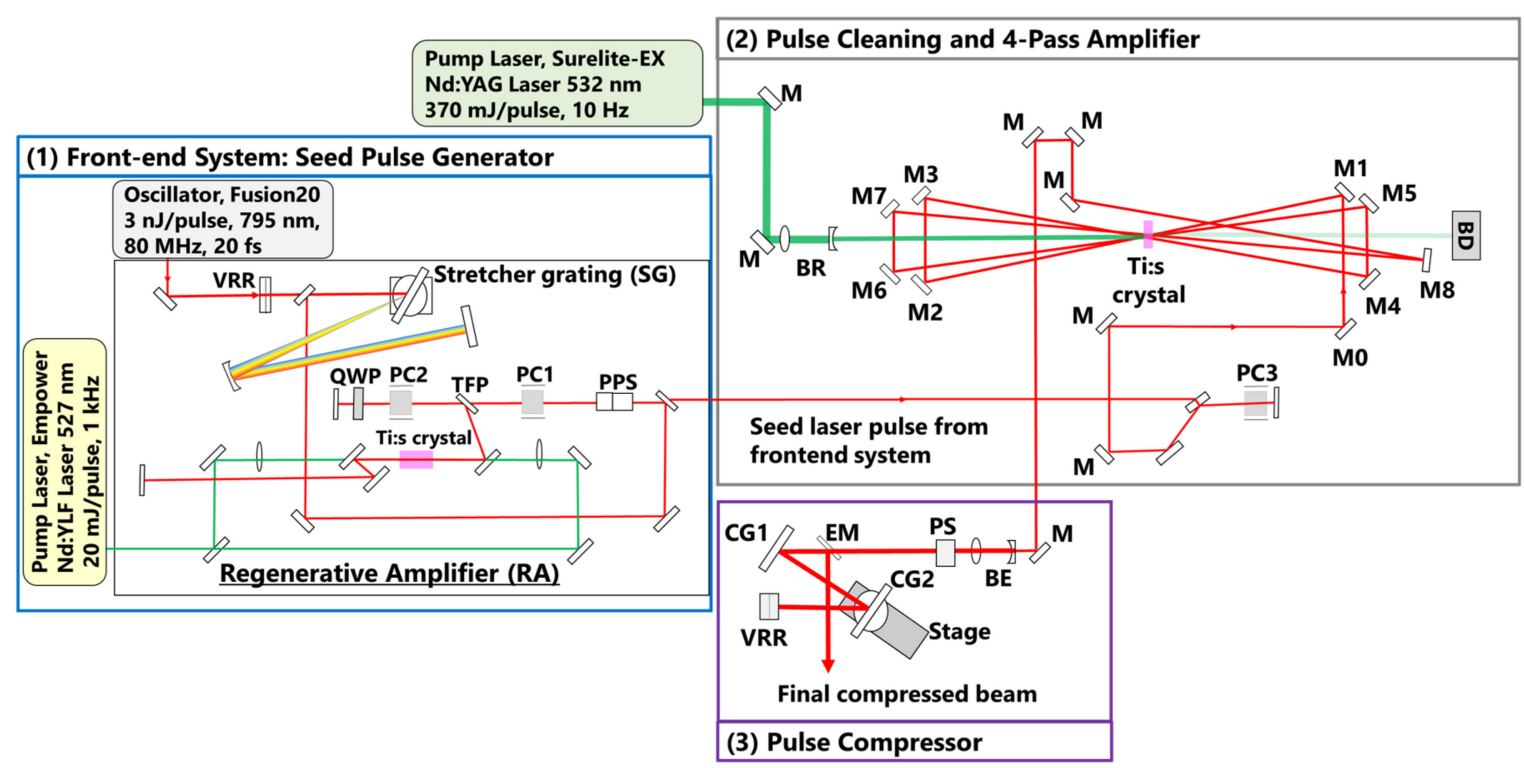

Figure 1. Schematic of the $1 \mathrm{TW} / 35$ fs Ti:sapphire laser amplifier system. VRR: vertical retro-reflector, PPS: polarization periscope, TFP: thin film polarizer, QWP: quarter-wave plate, M: mirror, BD: beam dump, BE: beam expander, BR: beam reducer, PS: periscope, PC: Pockels cell, CG: compressor grating, EM: exit mirror.

\subsection{4-pass Ti:sapphire Amplifier and Pulse Compression}

Figure 2 shows the measured output energies after the 4-pass (blue squares) amplifier when the pump beam energy changes. The result indicates that the maximum output pulse energy of $73 \mathrm{~mJ}$ can be obtained after 4-pass with an input pulse energy of $3.1 \mathrm{~mJ}$ and a pump beam energy of $370 \mathrm{~mJ}$, leading to an amplification factor of 23.5. Figure 2 also shows that the energy extraction efficiency is approximately $24 \%$.

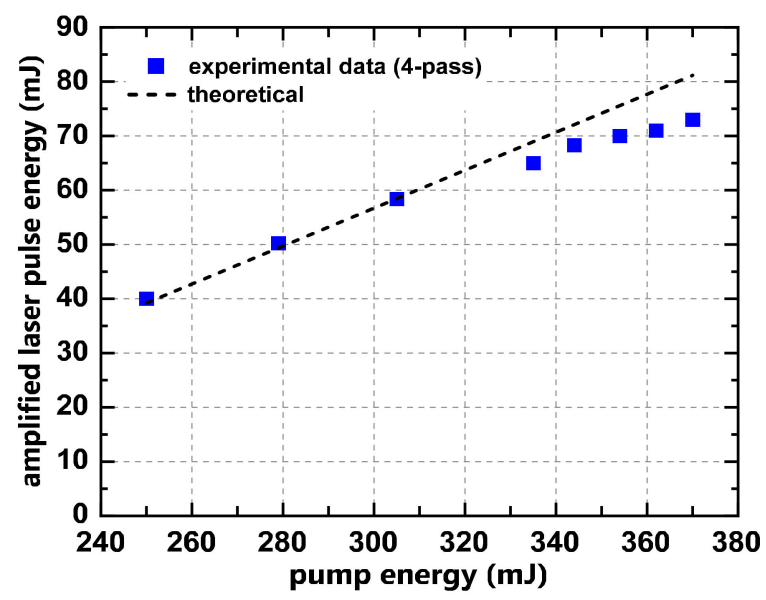

Figure 2. Measured laser pulse energy after 4-pass amplification (blue squares) and theoretical calculation using the Frantz-Nodvik equation [20,21] (black dotted line). The amplification efficiency (slope of the fitting line for the experimental data points) is $24 \%$.

The experimental result in Figure 2 can be compared with a theory. In the pulse amplification process, the amplified output pulse energy for a single pass can be given by a simple relation $E_{\text {out }}=E_{\text {in }} G_{0}$, where the input seed energy $E_{\text {in }}$ is amplified after passing through the amplifier, giving the amplified output energy $E_{\text {out }}$. Here, the 
small-signal gain $G_{0}$ is provided by $G_{0}=\exp \left(J_{\text {sto }} / J_{\text {sat }}\right)$, where the saturation fluence $J_{\text {sat }}$ is the intrinsic parameter of the Ti:sapphire $\left(\mathrm{Ti}: \mathrm{Al}_{2} \mathrm{O}_{3}\right)$ crystal $\left(1 \mathrm{~J} / \mathrm{cm}^{2}\right)[21,22]$ and $J_{\text {sto }}$ is the pump fluence stored in the gain medium. The pump fluence $J_{\text {sto }}$ stored in the medium can be deduced from experimental values from the expression given by $J_{\text {sto }}=\left(E_{p} / A\right) \eta_{Q D} \eta_{Q E} \eta_{A B S}$, where $E_{p}$ is the pump beam energy, $A$ is the cross-section of the pump beam, $\eta_{Q D}=\lambda_{\text {pump }} / \lambda_{\text {laser }}=532 \mathrm{~nm} / 800 \mathrm{~nm}$ is the quantum defect factor, $\eta_{Q E}$ is the quantum efficiency of the amplification process given by $\eta_{Q E}=0.81$ at room-temperature [23], $\eta_{A B S}$ is the experimentally measured absorption ratio of the pump laser energy in the crystal ( $\eta_{A B S}=0.92$ in our 2-cm-long Ti:sapphire crystal). Hence, $J_{\text {sto }}$ results in $0.933 \mathrm{~J} / \mathrm{cm}^{2}$ with the pump fluence of $1.88 \mathrm{~J} / \mathrm{cm}^{2}$. Here, it should be noted that $J_{\text {sto }}$ includes the effect of the medium length, which is included in $\eta_{A B S}$. Considering a back-of-envelope calculation here, the highest amplification factor in a single-pass is calculated as $G_{0}=\exp (0.933)=2.54$. More generally, the output fluence can be provided by the Frantz-Nodvik equation

$$
J_{\text {out }}=J_{\text {sat }} \ln \left\{1+G_{0}\left[\exp \left(\frac{J_{\text {in }}}{J_{\text {sat }}}\right)-1\right]\right\},
$$

where $J_{\text {in }}$ and $J_{\text {out }}$ are the input and output laser pulse fluences, respectively. The output pulse energy calculated from Equation (1) gives $7.9 \mathrm{~mJ}$ when the input laser energy in the amplifier is $3.1 \mathrm{~mJ}$. Equation (1) can be applied successively for multiple passes, meaning the output of one pass becomes the input for the next pass. In this way, the output energy of the 4-pass amplifier can be calculated, and the result is given by the dotted line in Figure 2. On the other hand, the experimentally measured energies after the 4-pass amplifier are represented by the blue squares in the same figure. Figure 2 shows that the output laser pulse energy is slightly lower than the calculated value for pump energy $>320 \mathrm{~mJ}$, which corresponds to a pump fluence of $1.63 \mathrm{~J} / \mathrm{cm}^{2}$. The deviation of the calculated result from the measured one in the figure may be due to the simple assumption of the Frantz-Nodvik theory, i.e., the theory is based on the assumption of uniform transverse distributions of the pump and seed laser beams, homogeneous broadening, and negligible loss in the system.

The beam profile of the amplified laser pulse for different output pulse energies was recorded using a beam profiler (OPHIR, model SP503U), as shown in Figure 3. From the figures, we can see the center of the output beam is saturated gradually as the output beam energy increases. The dimension of the beam with maximum output energy of $73 \mathrm{~mJ}$ is approximately $4.2 \mathrm{~mm} \times 5 \mathrm{~mm}$. The amplified laser pulse energy will have shot-to-shot fluctuations and the energy was measured to be $71.7 \pm 1.4 \mathrm{~mJ}(\mathrm{rms})$, as shown in Figure 4. The energy measurement also shows that the laser pulse energy from the 4-pass amplifier drifts very little with only $0.44 \mathrm{~mJ}$ over 10 minutes.

(a)

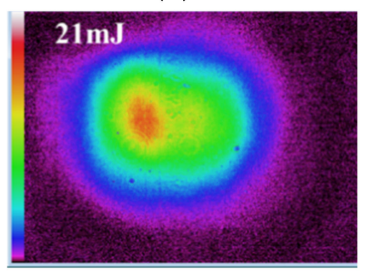

(b)

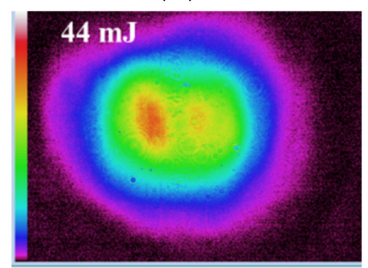

(c)

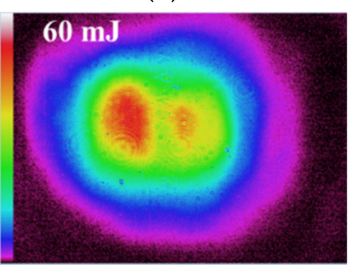

(d)

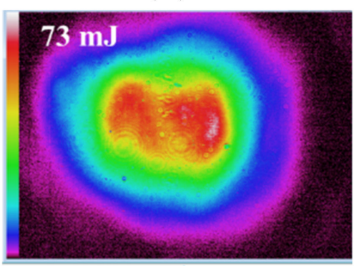

Figure 3. Beam profile of the amplified uncompressed laser pulse with different output energies: (a) $21 \mathrm{~mJ}$, (b) $44 \mathrm{~mJ}$, (c) $60 \mathrm{~mJ}$, and (d) $73 \mathrm{~mJ}$. The beam dimension for $73 \mathrm{~mJ}$ is approximately $4.2 \mathrm{~mm} \times 5.0 \mathrm{~mm}$ and the central part of the beam is almost saturated already in this high-energy case.

The amplified laser pulse from the 4-pass amplifier is sent to the pulse compressor consisting of two gratings and a vertical retro-reflector. The gratings were optimized for the shortest pulse, and the compressed beam properties were characterized by the gratingeliminated no-nonsense observation of ultrafast incident laser light e-fields (GRENOUILLE; 
Swamp Optics, model 8-20-USB) based on the frequency-resolved optical grating (FROG) technique [24-26]. Figure 5a shows the spectrum measured at three locations, where the compressed beam has a central wavelength of $800 \mathrm{~nm}$ and an FWHM bandwidth of $41.6 \mathrm{~nm}$. It should be noted here that the spectral bandwidth after 4-pass amplification did not change significantly by the gain-narrowing effect. It is important to preserve the compressible bandwidth throughout the amplification stages because the bandwidth determines the final laser pulse duration. The pulse duration after compression was measured to be 34 fs FWHM, as shown in Figure 5b.

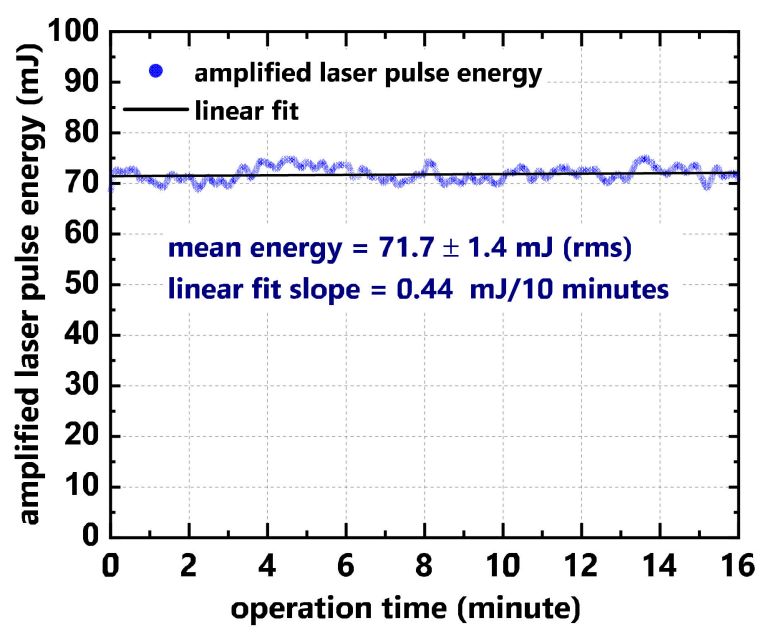

Figure 4. Measurement of the amplified laser pulse energy at a repetition rate of $10 \mathrm{~Hz}$ for more than $15 \mathrm{~min}$. The measured energy shows $71.7 \pm 1.4 \mathrm{~mJ}(\mathrm{rms})$, and the energy drift is $0.44 \mathrm{~mJ} / 10 \mathrm{~min}$.

(a)

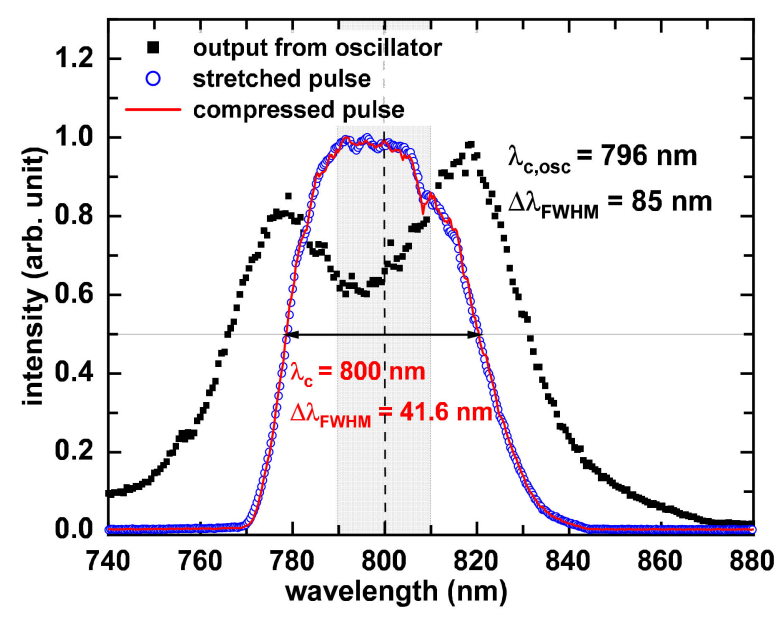

(b)

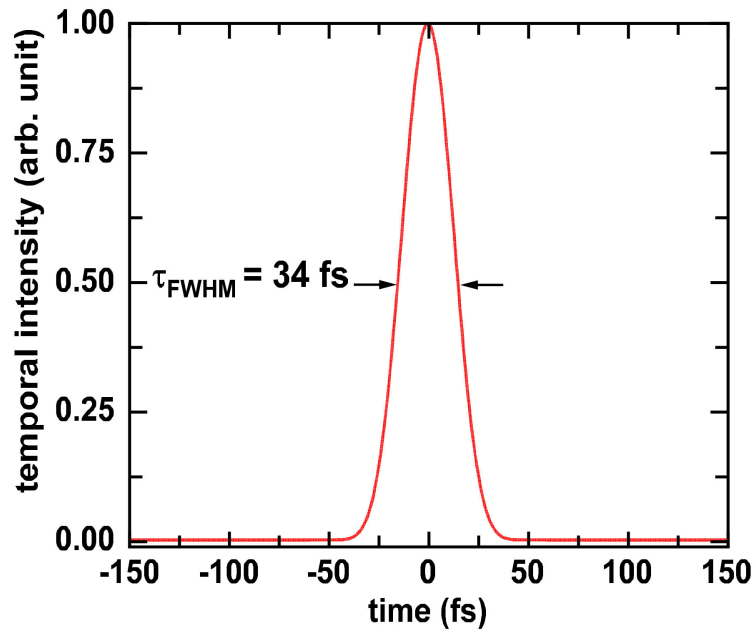

Figure 5. (a) Spectra of the laser pulse from the oscillator (black squares), the amplified stretched pulse after the 4-pass amplifier (blue circles) and the compressed laser pulse (red line). (b) Temporal intensity profile after compression, showing a pulse duration of $34 \mathrm{fs}$ FWHM.

The amplified spontaneous emission (ASE) of the compressed laser pulse was measured using a third-order cross-correlator (Amplitude Technologies, Sequoia). The temporal contrast ratio is defined as the intensity ratio $R$ of the peak intensity $I_{\text {peak }}$ to any prepulse or ASE/pedestal intensity $I_{A S E}$, i.e., $R=I_{\text {peak }} / I_{A S E}$ [27]. The temporal contrast ratio of our system was measured to be between $10^{7}$ and $10^{8}$, as shown in Figure 6. This contrast ratio is high compared with typical CPA laser contrast ratios that are about $10^{6}[27,28]$. The ps-prepulses were also measured, and they were found to be in the level of $10^{-4}$ or less. 
The maximum energy of the compressed laser pulse was $49.6 \mathrm{~mJ}$ when $73 \mathrm{~mJ}$ laser energy was sent to the pulse compressor, leading to a grating compression efficiency of $68 \%$. This is a reasonable result because we used rather old gratings.

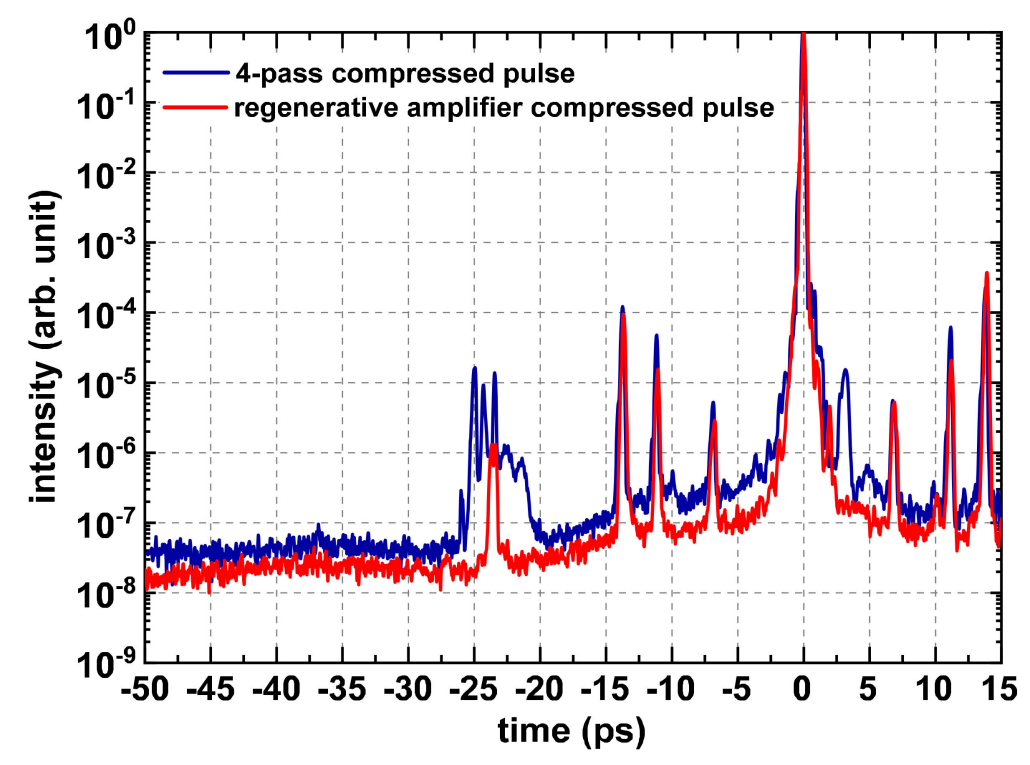

Figure 6. Amplified spontaneous emission (ASE) level of the compressed laser pulse measured by a third-order cross-correlator (Amplitude Technologies, Sequoia).

\section{Experimental Results for Two-Color-Based Intense THz Wave Generation Using the Laser Amplifier}

The generation of intense $\mathrm{THz}$ waves is a very important issue in science. Several ways were studied so far, and using a laser-produced plasma is a very promising way for high-energy THz wave generation. The two-color laser filamentation method [29-31], especially, is known to produce a strong $\mathrm{THz}$ pulse, where the fundamental and second harmonic laser pulses are focused together in the gas.

We used the developed laser amplifier system for the high-energy $\mathrm{THz}$ wave generation experiment, which employs the two-color laser filamentation method, as shown in Figure 7. The original laser pulse with $800 \mathrm{~nm}$ wavelength is focused in air through a beta-barium-borate (BBO) nonlinear crystal, generating a second harmonic laser pulse of $400 \mathrm{~nm}$ wavelength. Focusing the fundamental and second harmonic laser pulses in air ionizes the air molecules and produces plasma filamentation, leading to the generation of an intense $\mathrm{THz}$ pulse. The $\mathrm{THz}$ generation mechanism from the two-color method is attributed to the transient electric current in the laser-produced plasma, where mixing the fundamental and second harmonic laser pulses leads to an asymmetric net field in the plasma. The inserted image in Figure 7 shows an air plasma filament of about $30 \mathrm{~mm}$ in length, and the light is emitted mainly from nitrogen in the ultraviolet-visible range of bluish color [32].

In Figure 7, the produced $\mathrm{THz}$ pulse passes through the $0.5 \mathrm{~mm}$ thick silicon wafer that is optically transparent for the $\mathrm{THz}$ wave but blocks the laser pulses. The collimated $\mathrm{THz}$ pulse is then focused on the pyro-detector (Gentec-EO, model: THZ5B-MT) for energy measurement. The pyro-detector is removed later for THz-TDS diagnostics. The THz-TDS (also known as an electro-optic sampling (EOS) technique) is one of the most common $\mathrm{THz}$ diagnostic methods [33]. In this method, a ps-long $\mathrm{THz}$ pulse is sampled by a time-delayed fs-long probe pulse $(800 \mathrm{~nm})$, where the time delay relative to the $\mathrm{THz}$ pulse is scanned by a computer-controlled linear stage. When both the $\mathrm{THz}$ pulse and the probe laser pulse pass through the nonlinear ZnTe crystal [33-35], the polarization angle of the probe laser pulse changes by the birefringent $\mathrm{ZnTe}$ crystal in the THz field. This way, the electric field profile of the $\mathrm{THz}$ pulse can be measured. 


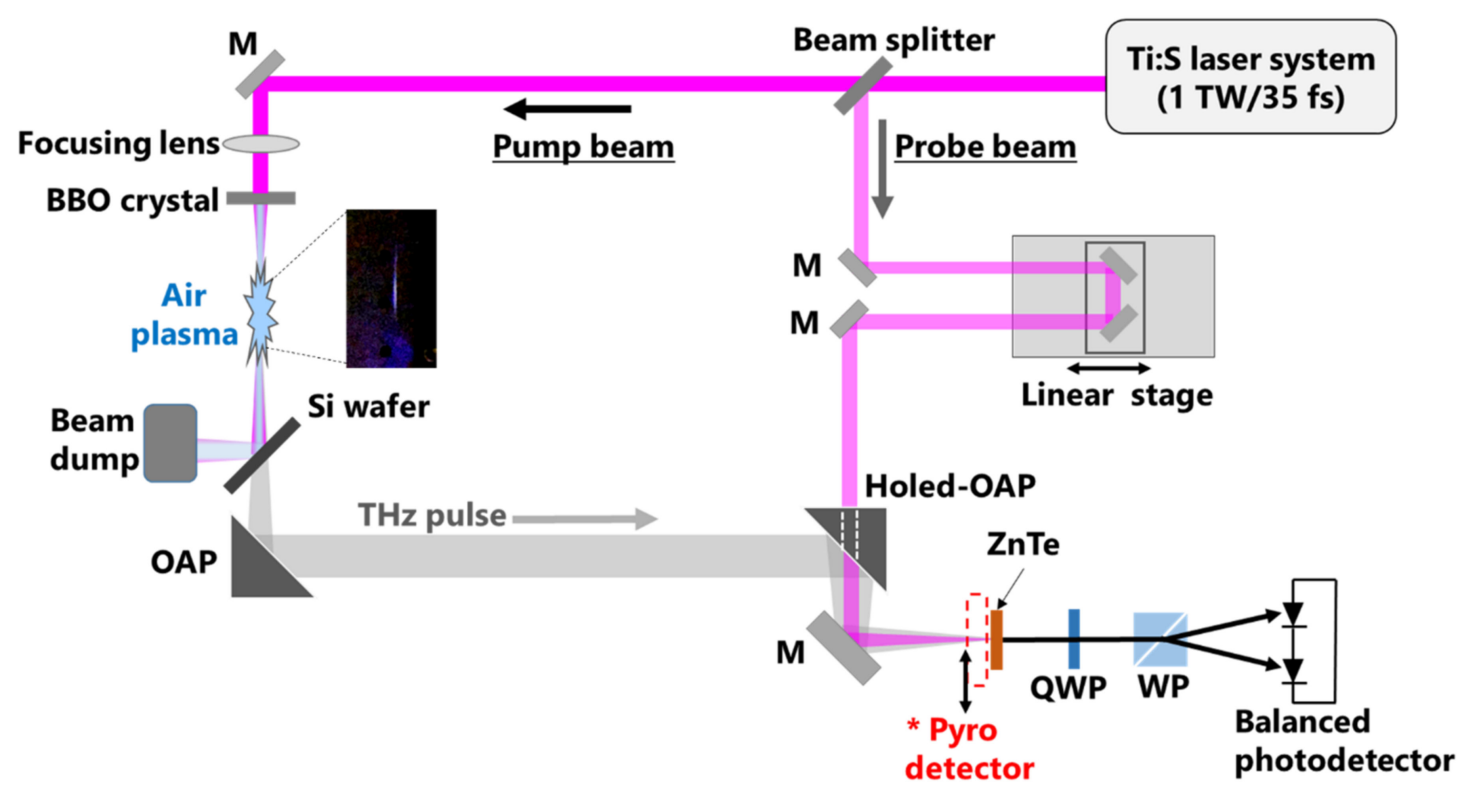

Figure 7. Schematic diagram of the experimental setup for $\mathrm{THz}$ wave generation using the two-color filamentation method and the THz-TDS (time-domain spectroscopy) diagnostics system. The pump beam is focused in the air to produce a strong $\mathrm{THz}$ pulse and the probe beam is sent to the THz-TDS system for diagnostics of the THz pulse. OAP: off-axis parabolic mirror, QWP: quarter-wave plate, WP: Wollaston prism, M: mirror. (* indicates retractable).

Figure 8a shows the THz pulse energy vs. the laser pulse energy (before frequency doubling), where the THz energy was before attenuation by the silicon wafer [36]. The experimental result shows that the maximum THz energy of $480 \mathrm{~nJ}$ can be obtained with the laser energy of $18.8 \mathrm{~mJ}$, which leads to the THz field strength of $242 \mathrm{kV} / \mathrm{cm}$. This result yields a $\mathrm{THz}$ conversion efficiency of $2.5 \times 10^{-5}$. It seems that the THz energy from our experiment is quite reasonable compared with other results [31,37]. Figure 8a also shows that the THz energy increases sharply in the beginning as the laser energy increases, but it is gradually saturated. Figure $8 \mathrm{~b}$ shows the THz pulse form (in the inset) measured by the THz-TDS method and its Fourier transformation, yielding a spectrum of the THz pulse. It should be noted that the spectrum of the THz pulse from THz-TDS has a cut-off frequency around $5 \mathrm{THz}$. This frequency bandwidth is due to the limitation of the phonon-resonance properties of the ZnTe crystal [38].

As Figure 8a shows, the THz pulse energy is saturated, and it does not increase anymore in the high laser energy regime. This saturation phenomenon has not been explained by any rigorous theory or simulation so far. To investigate this phenomenon seriously, therefore, we performed a series of two-dimensional (2D) particle-in-cell (PIC) simulations. For the PIC simulations, we used the EPOCH code [39] to model the interaction between the laser pulse and neutral gas/plasma, where a fully electromagnetic, relativistic, and self-consistent theory is employed. The simulation window of $200 \mu \mathrm{m} \times 300 \mu \mathrm{m}$ in size is a non-moving spatial domain which is discretized by $4000 \times 750$ cells with two particles per cell. For simplicity, neutral nitrogen species with an atmospheric density of $n_{N}=2.68 \times 10^{19} \mathrm{~cm}^{-3}$ was used in the simulation. We used linearly polarized Gaussian laser pulses with wavelengths of $800 \mathrm{~nm}$ and $400 \mathrm{~nm}$ and a pulse duration of $35 \mathrm{fs}$ (FWHM). The laser pulses are focused on a focal plane with a beam waist of $w_{0}=10 \mu \mathrm{m}$. Figure 9 shows the PIC simulation result, i.e., 2D plots of the $\mathrm{THz}$ wave for different laser pulse energies, implying that a stronger $\mathrm{THz}$ pulse is generated by a higher laser pulse energy. The $\mathrm{THz}$ pulse energy can be estimated from the simulation result (open circle) and compared with the experimental result (blue square) in Figure 8a. Here, it should be noted that the THz pulse energy from the PIC simulation result is also saturated in the high laser energy regime, which is consistent with the experimental result. This phenomenon can be understood by the fact that the plasma filament length, which is a critical factor 
in $\mathrm{THz}$ energy, does not increase linearly as the laser pulse energy increases. In fact, two factors play an important role in the formation of the laser filament when an intense laser pulse propagates in gas. One is the Kerr effect that provides a focusing force for the laser, and the other is a defocusing effect by the ionization-induced diffraction in addition to the ordinary diffraction. As the laser energy increases, more neutral atoms in the air are ionized, and the Kerr effect is reduced naturally, while the ionization-induced diffraction effect will be more dominant. Hence, the filament length can be limited although the laser energy increases, leading to the saturation in THz energy. Here, it should be noted that the relativistic self-focusing effect [40] does not have to be taken into account as it is too small in our case. The calculation with our laser and plasma parameters in this experiment shows that the normalized vector potential of the focused laser beam is $a_{0}<1$ and the peak laser power is less than the critical power $P_{c}$ for relativistic self-focusing, where $a_{0}$ and $P_{c}$ are given by $\left.a_{0} \cong 0.85 \times 10^{-9} \sqrt{I\left[\mathrm{~W} / \mathrm{cm}^{2}\right.}\right] \lambda[\mu \mathrm{m}]$ and $P_{c}[\mathrm{GW}]=17.4\left(\omega / \omega_{p}\right)^{2}$ in practical units, respectively [40]. Here, $I$ is the focused laser beam intensity, $\lambda$ is the laser wavelength, $\omega$ and $\omega_{p}$ are the laser and plasma frequencies, respectively.

(a)

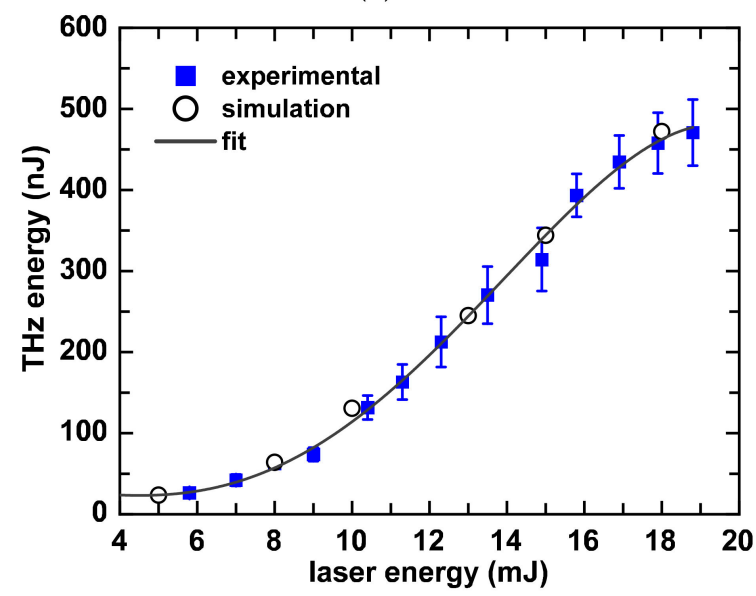

(b)

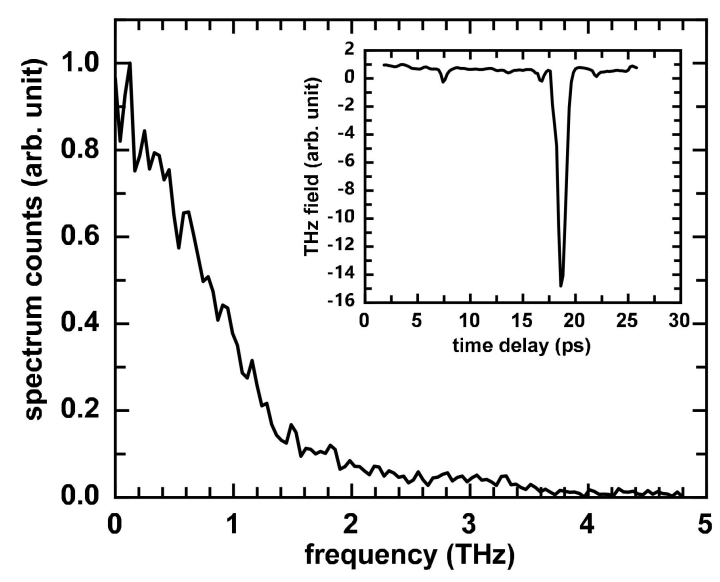

Figure 8. (a) Measured THz pulse energy as a function of the laser energy (before frequency doubling). The simulation result (open circle) is normalized to the experimental result (blue square) for comparison. (b) Spectrum of the THz pulse obtained from Fast-Fourier Transform (FFT) of the THz pulse in the inset, where the THz pulse was directly measured from the THz-TDS method. Note that the spectrum is limited up to a maximum frequency of $\sim 5 \mathrm{THz}$ due to the intrinsic properties of the ZnTe crystal.

(a) $\mathrm{E}=5 \mathrm{~mJ}$

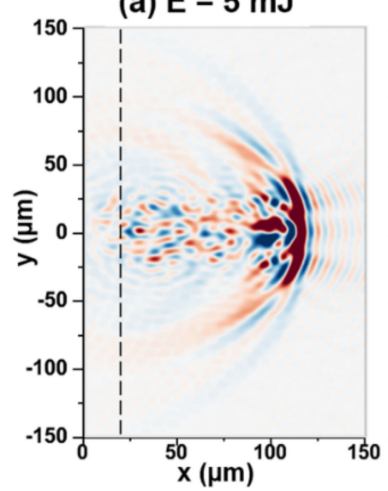

(b) $E=10 \mathrm{~mJ}$

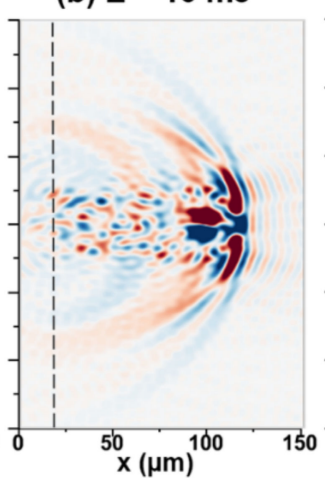

(c) $E=15 \mathrm{~mJ}$

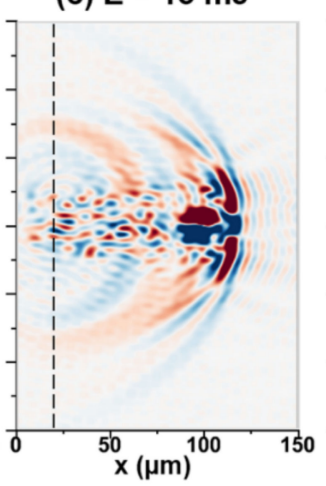

(d) $\mathrm{E}=\mathbf{2 0 \mathrm { mJ }}$

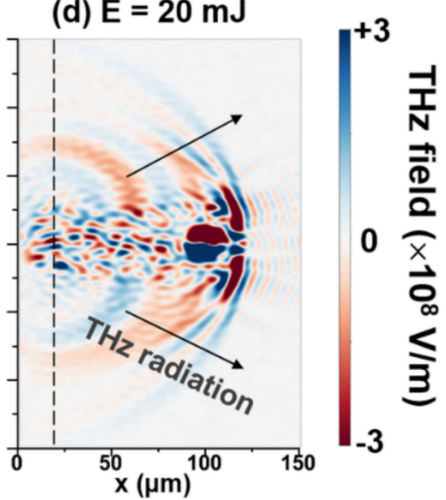

Figure 9. 2D PIC simulation results for THz pulse generation by the two-color method: 2D-plot of the THz electric field strength with a different laser pulse energy of (a) $5 \mathrm{~mJ}$, (b) $10 \mathrm{~mJ}$, (c) $15 \mathrm{~mJ}$, and (d) $20 \mathrm{~mJ}$. The result shows that a stronger $\mathrm{THz}$ wave is generated by a higher laser pulse energy. In the figures, the black dashed line indicates the focal plane of the laser pulse, and the black arrows indicate the $\mathrm{THz}$ wave propagation. 


\section{Conclusions}

In conclusion, we successfully developed a TW-class high-power amplifier system using some existing parts in our laboratory, which consists of a mode-locked oscillator, a regenerative amplifier, and a 4-pass Ti:sapphire amplifier. The CPA-based laser amplifier was characterized, and it was found to deliver an energy of $49.6 \mathrm{~mJ} /$ pulse and a pulse duration of $34 \mathrm{fs}$, resulting in more than $1 \mathrm{TW}$ in power. The ASE and ps-prepulse levels were measured to be quite low, i.e., less than $10^{-7}$ and $10^{-4}$, respectively. We used this TW-class Ti:sapphire laser amplifier to generate an intense $\mathrm{THz}$ wave by focusing the fundamental and second harmonic laser pulses in the air. It was found that the $\mathrm{THz}$ conversion efficiency is on the order of $10^{-5}$, and the THz pulse energy is saturated as the laser energy increases. Fully electromagnetic, relativistic, and self-consistent PIC simulations were performed for comparison with the experimental result, and it turned out that both the simulation and experimental results show a similar saturation phenomenon.

Author Contributions: H.S. conceived the idea. V.L.J.P., S.J., K.K., and J.K. prepared the laser amplifier development and characterization. K.K., S.J., and K.R. prepared and performed the THz experiment. V.L.J.P. performed the 2D-PIC simulation, data analysis, and wrote the draft of the manuscript. All authors participated in preparatory discussions and manuscript improvement. All authors have read and agreed to the published version of the manuscript.

Funding: This research was funded by the National Research Foundation of Korea (NRF, Grant \#: 2017R1A2B3010765).

Data Availability Statement: The data presented in this paper are available on request from the corresponding author.

Acknowledgments: The authors acknowledge H.J. Lee and H.I. Kim in the group for their technical supports. The EPOCH code was developed under the UK EPSRC grants EP/G054950/1, $\mathrm{EP} / \mathrm{G} 056803 / 1, \mathrm{EP} / \mathrm{G} 055165 / 1$, and EP/M022463/1.

Conflicts of Interest: The authors declare no conflict of interest.

\section{References}

1. Maiman, T.H. Stimulated optical radiation in ruby. Nature 1960, 187, 493-494. [CrossRef]

2. Sixty years of lasers. Nat. Rev. Phys. 2020, 2, 221. [CrossRef]

3. Strickland, D.; Mourou, G. Compression of amplified chirped optical pulses. Opt. Commun. 1985, 55, 447-449. [CrossRef]

4. Yoon, J.W.; Jeon, C.; Shin, J.; Lee, S.K.; Lee, H.W.; Choi, I.W.; Kim, H.T.; Sung, J.H.; Nam, C.H. Achieving the laser intensity of $5.5 \times 10^{22} \mathrm{~W} / \mathrm{cm}^{2}$ with a wavefront-corrected multi-PW laser. Opt. Express 2019, 27, 20412-20420. [CrossRef]

5. Tanaka, K.A.; Spohr, K.M.; Balabanski, D.L.; Balascuta, S.; Capponi, L.; Cernaianu, M.O.; Cuciuc, M.; Cucoanes, A.; Dancus, I.; Dhal, A.; et al. Current status and highlights of the ELI-NP research program. Matter Radiat. Extremes 2020, 5, 024402. [CrossRef]

6. Gonsalves, A.J.; Nakamura, K.; Daniels, J.; Benedetti, C.; Pieronek, C.; de Raadt, T.C.H.; Steinke, S.; Bin, J.H.; Bulanov, S.S.; van Tilborg, J.; et al. Petawatt laser guiding and electron beam acceleration to $8 \mathrm{GeV}$ in a laser-heated capillary discharge waveguide. Phys. Rev. Lett. 2019, 122, 084801. [CrossRef]

7. Ke, L.; Yu, C.; Feng, K.; Qin, Z.; Jiang, K.; Wang, H.; Luan, S.; Yang, X.; Xu, Y.; Leng, Y.; et al. Optimization of electron beams based on plasma-density modulation in a laser-driven wakefield accelerator. Appl. Sci. 2021, 11, 2560. [CrossRef]

8. Leemans, W.P.; Gonsalves, A.J.; Mao, H.S.; Nakamura, K.; Benedetti, C.; Schroeder, C.B.; Tóth, C.; Daniels, J.; Mittelberger, D.E.; Bulanov, S.S.; et al. Multi-GeV electron beams from capillary-discharge-guided subpetawatt laser pulses in the self-trapping regime. Phys. Rev. Lett. 2014, 113, 245002. [CrossRef]

9. Polz, J.; Robinson, A.P.L.; Kalinin, A.; Becker, G.A.; Fraga, R.A.C.; Hellwing, M.; Hornung, M.; Keppler, S.; Kessler, A.; Klöpfel, D.; et al. Efficient laser-driven proton acceleration from a cccryogenic solid hydrogen target. Sci. Rep. 2019, 9, 16534. [CrossRef]

10. Bagchi, S.; Tayyab, M.; Pasley, J.; Robinson, A.P.L.; Nayak, M.; Chakera, J.A. Quasi mono-energetic heavy ion acceleration from layered targets. Phys. Plasmas 2021, 28, 023108. [CrossRef]

11. Zhu, X.-L.; Chen, M.; Weng, S.-M.; Yu, T.-P.; Wang, W.-M.; He, F.; Sheng, Z.-M.; McKenna, P.; Jaroszynski, D.A.; Zhang, J. Extremely brilliant $\mathrm{GeV} \gamma$-rays from a two-stage laser-plasma accelerator. Sci. Adv. 2020, 6, 7240. [CrossRef] [PubMed]

12. Kozlova, M.; Andriyash, I.; Gautier, J.; Sebban, S.; Smartsev, S.; Jourdain, N.; Chulagain, U.; Azamoum, Y.; Tafzi, A.; Goddet, J.-P.; et al. Hard X-rays from laser-wakefield accelerators in density tailored plasmas. Phys. Rev. X 2020, 10, 011061. [CrossRef] 
13. Kim, K.Y.; Taylor, A.J.; Glownia, J.H.; Rodriguez, G. Coherent control of terahertz supercontinuum generation in ultrafast laser-gas interactions. Nat. Photonics 2008, 2, 605-609. [CrossRef]

14. He, Z.-H.; Thomas, A.G.R.; Beaurepaire, B.; Nees, J.A.; Hou, B.; Malka, V.; Krushelnick, K.; Faure, J. Electron diffraction using ultrafast electron bunches from a laser-wakefield accelerator at $\mathrm{kHz}$ repetition rate. Appl. Phys. Lett. 2013, 102, 064104. [CrossRef]

15. Bonvalet, A.; Darmon, A.; Lambry, J.C.; Martin, J.L.; Audebert, P. 1 kHz tabletop ultrashort hard X-ray source for time-resolved X-ray protein crystallography. Opt. Lett. 2006, 31, 2753-2755. [CrossRef]

16. Raksi, F.; Wilson, K.R.; Jiang, Z.M.; Ikhlef, A.; Cote, C.Y.; Kieffer, J.C. Ultrafast X-ray absorption probing of a chemical reaction. J. Chem. Phys. 1996, 104, 6066-6069. [CrossRef]

17. Tonouchi, M. Cutting-edge terahertz technology. Nat. Photonics 2007, 1, 97-105. [CrossRef]

18. Dhillon, S.S.; Vitiello, M.S.; Linfield, E.H.; Davies, A.; Hoffmann, M.; Booske, J.; Paoloni, C.; Gensch, M.; Weightman, P.; Williams, G.P.; et al. The 2017 terahertz science and technology roadmap. J. Phys. D Appl. Phys. 2017, 50, 043001. [CrossRef]

19. Treacy, E. Optical pulse compression with diffraction gratings. IEEE J. Quantum Electron. 1969, 5, 454-458. [CrossRef]

20. Frantz, L.M.; Nodvik, J.S. Theory of pulse propagation in a laser amplifier. J. Appl. Phys. 1963, 34, 2346-2349. [CrossRef]

21. Salin, F. How to Manipulate and Change the Characteristics of Laser Pulses. In Femtosecond Laser Pulses, 2nd ed.; Rulliere, C., Ed.; Springer Science Business Media, Inc.: New York, NY, USA, 2005; pp. 175-194. [CrossRef]

22. Perry, M.D.; Mourou, G. Terawatt to Petawatt subpicosecond lasers. Science 1994, 264, 917. [CrossRef]

23. Moulton, P.F. Spectroscopic and laser characteristics of Ti: $\mathrm{Al}_{2} \mathrm{O}_{3}$. J. Opt. Soc. Am. B 1986, 3, 125-133. [CrossRef]

24. Kane, D.J.; Trebino, R. Characterization of arbitrary femtosecond pulses using frequency resolved optical gating. IEEE J. Quantum Electron. 1993, 29, 571-579. [CrossRef]

25. Kane, D.J.; Taylor, A.J.; Trebino, R.; DeLong, K.W. Single-shot measurement of the intensity and phase of a femtosecond UV laser pulse using frequency-resolved optical gating. Opt. Lett. 1994, 19, 1061-1063. [CrossRef]

26. Trebino, R.; DeLong, K.W.; Fittinghoff, D.N.; Sweetser, J.N.; Krumbügel, M.A.; Kane, D.J. Measuring ultrashort laser pulses in the time-frequency domain using frequency-resolved optical gating. Rev. Sci. Instrum. 1997, 68, 3277-3295. [CrossRef]

27. Fourmaux, S.; Payeur, S.; Buffechoux, S.; Lassonde, P.; St-Pierre, C.; Martin, F.; Kieffer, J.C. Pedestal cleaning for high laser pulse contrast ratio with a 100 TW class laser system. Opt. Express 2011, 19, 8486-8497. [CrossRef]

28. McKenna, P.; Lindau, F.; Lundh, O.; Neely, D.; Persson, A.; Wahlström, C.-G. High-intensity laser-driven proton acceleration: Influence of pulse contrast. Philos. Trans. Royal Soc. A 2006, 364, 711-723. [CrossRef]

29. Cook, D.J.; Hochstrasser, R.M. Intense terahertz pulses by four-wave rectification in air. Opt. Lett. 2000, 25, 1210-1212. [CrossRef]

30. Kress, M.; Löffler, T.; Eden, S.; Thomson, M.; Roskos, H.G. Terahertz-pulse generation by photoionization of air with laser pulses composed of both fundamental and second-harmonic waves. Opt. Lett. 2004, 29, 1120-1122. [CrossRef]

31. Kim, K.-Y.; Glownia, J.H.; Taylor, A.J.; Rodriguez, G. Terahertz emission from ultrafast ionizing air in symmetry-broken laser fields. Opt. Express 2007, 15, 4577-4584. [CrossRef] [PubMed]

32. Dandl, T.; Heindl, T.; Ulrich, A. Fluorescence of nitrogen and air. J. Instrum. 2012, 7, P11005. [CrossRef]

33. Gallot, G.; Grischkowsky, D. Electro-optic detection of terahertz radiation. J. Opt. Soc. Am. B 1999, 16, 1204-1212. [CrossRef]

34. Jang, D.; Uhm, H.S.; Jang, D.; Hur, M.S.; Suk, H. Electron density characterization of inductively-coupled argon plasmas by the terahertz time-domain spectroscopy. Plasma Sources Sci. Technol. 2016, 25, 065008. [CrossRef]

35. Neu, J.; Schmuttenmaer, C.A. Tutorial: An introduction to terahertz time domain spectroscopy (THz-TDS). J. Appl. Phys. 2018, 124, 231101. [CrossRef]

36. Kaltenecker, K.J.; Kelleher, E.J.R.; Zhou, B.; Jepsen, P.U. Attenuation of THz beams: A “how to" tutorial. J. Infrared Millim. Terahertz Waves 2019, 40, 878-904. [CrossRef]

37. Blanchard, F.; Razzari, L.; Bandulet, H.-C.; Sharma, G.; Morandotti, R.; Kieffer, J.-C.; Ozaki, T.; Reid, M.; Tiedje, H.; Haugen, H. Generation of $1.5 \mu \mathrm{J}$ single-cycle terahertz pulses by optical rectification from a large aperture ZnTe crystal. Opt. Express 2007, 15, 13212-13220. [CrossRef] [PubMed]

38. Gallot, G.; Zhang, J.; McGowan, R.W.; Jeon, T.-I.; Grischkowsky, D. Measurements of the THz absorption and dispersion of ZnTe and their relevance to the electro-optic detection of THz radiation. Appl. Phys. Lett. 1999, 74, 3450-3452. [CrossRef]

39. Arber, T.D.; Bennett, K.; Brady, C.S.; Lawrence-Douglas, A.; Ramsay, M.G.; Sircombe, N.J.; Gillies, P.; Evans, R.G.; Schmitz, H.; Bell, A.R.; et al. Contemporary particle-in-cell approach to laser-plasma modelling. Plasma Phys. Control. Fusion 2015, $57,113001$. [CrossRef]

40. Esarey, E.; Sprangle, P.; Krall, J.; Ting, A. Self-focusing and guiding of short laser pulses in ionizing gases and plasmas. IEEE J. Quantum Electron. 1997, 33, 1879-1914. [CrossRef] 